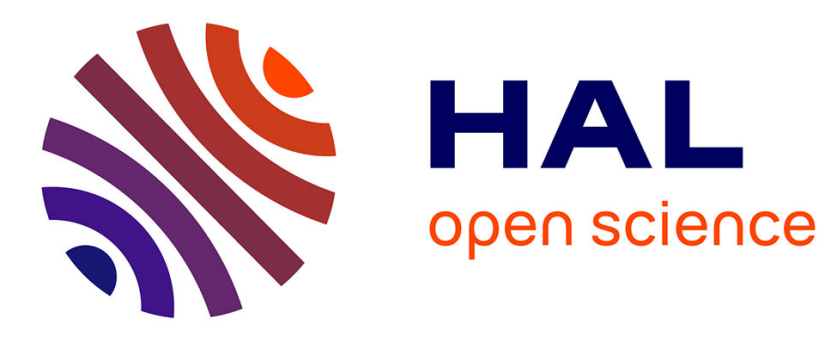

\title{
Pipeline tracking for fully-actuated autonomous underwater vehicle using visual servo control
}

Szymon Krupinsky, Guillaume Allibert, Minh Duc Hua, Tarek Hamel

\section{To cite this version:}

Szymon Krupinsky, Guillaume Allibert, Minh Duc Hua, Tarek Hamel. Pipeline tracking for fullyactuated autonomous underwater vehicle using visual servo control. American Control Conference (ACC12), Jun 2012, Montreal, Canada. pp.6196-6202. hal-00716751

\section{HAL Id: hal-00716751 \\ https://hal.science/hal-00716751}

Submitted on 11 Jul 2012

HAL is a multi-disciplinary open access archive for the deposit and dissemination of scientific research documents, whether they are published or not. The documents may come from teaching and research institutions in France or abroad, or from public or private research centers.
L'archive ouverte pluridisciplinaire HAL, est destinée au dépôt et à la diffusion de documents scientifiques de niveau recherche, publiés ou non, émanant des établissements d'enseignement et de recherche français ou étrangers, des laboratoires publics ou privés. 


\title{
Pipeline tracking for fully-actuated autonomous underwater vehicle using visual servo control
}

\author{
Szymon Krupiński*† Guillaume Allibert* \\ Minh-Duc Huab \\ Tarek Hamel* \\ szymon.krupinski@cybernetix.fr allibert@i3s.unice.fr \\ huaeisir.upmc.fr thamelei3s.unice.fr
}

\begin{abstract}
This paper describes a nonlinear image-based visual servo control algorithm for the pipeline tracking problem of a fully-actuated underwater vehicle. The dynamic model of a generic autonomous underwater vehicle (AUV), incorporating all significant forces and torques is developed and a generic velocity control strategy is proposed. The desired velocities in the plane orthogonal to the direction of the pipeline along with the yaw velocity are derived from the image sequence using bi-normalised Plücker coordinates of the pipeline borders. The desired velocity along the pipeline is specified by the operator and finally the desired velocities in pitch and roll angles are set to zero. The asymptotic stability of the visual servo control strategy is proved and simulation testing is carried out to show performance of the control approach.
\end{abstract}

\section{INTRODUCTION}

The application of Autonomous Underwater Vehicles (AUVs) has improved the accessibility of scientific and industrial data in several domains. From the initial goals of data gathering in vast open spaces, the missions of these vehicles are now expected to be executed in close proximity of a complex sea bottom or submarine structures, given that it can create revolutionary applications of AUVs in subsea inspection and maintenance. The shift from simple inertial navigation to sensor-referenced and model-based control is prompting the development of new techniques. Proven robustness and steady performance in the face of often noisy data is a requirement for assuring safe operation of these vehicles, often very expensive.

The main challenging problem when dealing with AUV is inherently linked to the nonlinear dynamics of the system and highly coupled in 6 d.o.f. The interaction with a dense medium (water) has measurable effects due to friction and dynamic surface pressure, bringing in the terms of added mass and damping. There are several formulations of the dynamics of an underwater vehicle taking all these effects into consideration, notably by Fossen [1], Bailey et al. [2] and Leonard [3], each of them with different merits and certain considerations. The latter reference, based on the change of momentum expression, provides good insight into the energy of the system.

There is a vast array of designs and control system architectures for AUVs, well summarised by Yuh in [4]. Typically, AUVs carry inertial sensors to estimate accelerations. The estimation, inherently burdened by drift error, is improved

* I3S UNS-CNRS, Sophia Antipolis, France

b ISIR UPMC-CNRS, Paris, France

† Cybernetix SA, Technopôle de Château-Gombert, 306 rue Albert Einstein, Marseille, France by exploiting the measurements of a magnetic compass along with a Doppler Velocity Log (DVL) which measures linear velocities w.r.t the surrounding medium. An underwater vehicle usually uses a sonar (scanning, multi-beam or sidescan) or a video camera to sense its environment. Although far from Global Positioning System (GPS) in performance, there exist systems which allows positioning with respect to some locally fixed beacons. Most vehicles share a couple of types of common physical dynamics and similar tasks. The control problem thus remains a universal and shared core for most of the academic centers researching AUVs.

Results in non-linear control of AUV systems have been achieved using a host of different techniques. Amongst the approaches which exploit the nonlinearities are techniques of direct Lyapunov control [5], sliding mode control [6], adaptive control (LQ [7], H- $\infty$ [8]), optimal control [9] and a host of linearizing methods. Moreover, some developments with respect to the sensor-based navigation, centered mostly around sonar-based tracking and simultaneous localisation and mapping (SLAM) have been recently proposed [10]. Notable examples of work where the sensor input is used for structure-referenced navigation are [11] and [12], while [13] and [14] focus on the task of pipeline tracking, with little regards to the nonlinearity of the control problem.

While almost all techniques developed previously are based on the reconstruction of the relative position of the vehicle in its environment, this paper focuses on an imagebased visual servoing (IBVS). The advantage of IBVS schemes over position-based visual servoing (PBVS) relies on the fact that the relative position of the robot with respect to its environment is not required and only 2D image features are needed. Hence, IBVS techniques are usually more suitable for real-time algorithms since they exploit geometrical properties of the imaging systems and their relations to the environment/robot. The image features considered are the bi-normalised Plücker coordinates [15] of the pipeline borders. The control design is split into two parts: an inner-loop controller to stabilise the velocities of the vehicle around a desired set point and an outer-loop to derive the velocity desired set point from image features.

The paper is organized as follows: Section II presents the developed dynamical system modelling an AUV. Section III describes the chosen control problem along with the visual features considered. Section IV derives the inner-loop controller and analyses the stability of the system. Section V describe the velocity desired set point from image features. Section V-B analyses the stability of the full system. Section 
VI applies the control strategy to a realistic model of an underwater vehicle and exhibits some simulation results. The final section provides a short conclusion and an indication of future works.

\section{SySTEM MODELING}

The following conventions of the reference frames are used: $\mathcal{A}=\left\{e_{1}^{a}, e_{2}^{a}, e_{3}^{a}\right\}$ is the inertial reference frame and $\mathcal{B}=\left\{e_{1}^{b}, e_{2}^{b}, e_{3}^{b}\right\}$ represents the body-fixed frame. The position of the body-fixed frame in $\mathcal{A}$ is the center of buoyancy (CB) and denoted $b_{B} \in \mathcal{A}$. The center of gravity (CG) is denoted $b_{G} \in \mathcal{A}$ and $r_{G} \in \mathcal{B}$ (see Fig. 1). The orientation of $\mathcal{B}$ with respect to $\mathcal{A}$ is denoted $R \in S O(3), \mathcal{B} \mapsto \mathcal{A}$, an element of the special orthogonal group. The remaining state variables of the system are the velocities: linear $V$ and angular $\Omega$, both expressed in $\mathcal{B}$. Another important reference frame is bound to the camera: $\mathcal{C}=\left\{e_{1}^{c}, e_{2}^{c}, e_{3}^{c}\right\}$ which is displaced from the origin of $\mathcal{B}$ by a vector $r_{C}$, keeping its base vectors parallel to those of $\mathcal{B}$. Let $\left\{e_{1}, e_{2}, e_{3}\right\}$ denote the canonical basis of $\mathbb{R}^{3}$. Define:

$$
\nu=\left[\begin{array}{l}
V \\
\Omega
\end{array}\right]=\left[\begin{array}{llllll}
u & v & w & p & q & r
\end{array}\right]^{T}=\left[\begin{array}{c}
\text { surge } \\
\text { sway } \\
\text { heave } \\
\text { rill } \\
\text { pitch } \\
\text { yaw }
\end{array}\right]
$$

Commonly used parameters of a rigid body are the total mass $m$ and the moment of inertia tensor $I_{0}$.

When characterised at an arbitrary point, for instance $C B$ $(C B \neq C G)$, the kinetic energy of the system is given by ${ }^{1}$ :

$$
E_{R B}=\frac{1}{2} \nu^{T}\left[\begin{array}{cc}
m I_{3 \times 3} & -m r_{G \times} \\
m r_{G \times} & I_{0}
\end{array}\right] \nu=\frac{1}{2} \nu^{T} M_{R B} \nu
$$

where $r_{G}$ is the position of the center of gravity (CG) in the body-fixed frame with the origin at $C B$.

According to Kirchhoff and Lamb theory [16], the kinetic energy of the liquid surrounding the body is given by [3]:

$$
E_{A}=\frac{1}{2} \nu^{T} M_{A} \nu=\frac{1}{2} \nu^{T}\left[\begin{array}{ll}
M_{A}^{11} & M_{A}^{12} \\
M_{A}^{21} & M_{A}^{22}
\end{array}\right] \nu
$$

where $M_{A}$ is known as the added mass matrix. Consequently, the total of the kinetic energy of the body in its medium is:

$$
E_{T}=E_{R B}+E_{A}=\frac{1}{2} \nu^{T} M_{T} \nu
$$

where $M_{T}=\left[\begin{array}{cc}M & M_{T}^{12} \\ M_{T}^{21} & I\end{array}\right]=\left[\begin{array}{cc}m I_{3 \times 3}+M_{A}^{11} & -m r_{G \times}+M_{A}^{12} \\ m r_{G \times}+M_{A}^{21} & I_{0}+M_{A}^{22}\end{array}\right]$

All vehicles with three mutually perpendicular axes of symmetry (em i.e. ellipsoid, box-shaped, ...) approximatively have a diagonal form for the added mass matrix $M_{A}$. In practice, the diagonal terms of $M_{A}$ are often significantly larger that the other terms, and therefore the matrix $M_{A}$ can be approximated by a diagonal matrix [1]. Consequently, $M_{T}^{21}=-M_{T}^{12}=D:=m r_{G \times}$.

Recalling the total kinetic energy of the body-liquid system (1), one can derive the translational $(P)$ and rotational momentums (П) as follows [3]:

$$
\begin{aligned}
& P=\frac{\partial E_{T}}{\partial V}=M V+D^{T} \Omega \\
& \Pi=\frac{\partial E_{T}}{\partial \Omega}=I \Omega+D V
\end{aligned}
$$

\footnotetext{
${ }^{1}$ The notation $\vec{v}_{\times}=\left[\begin{array}{ccc}0 & -v_{3} & v_{2} \\ v_{3} & 0 & -v_{1} \\ -v_{2} & v_{1} & 0\end{array}\right]$ is used to abbreviate the skewsymmetric matrix multiplicative representation of vector product.
}

According to [3] and [17], the dynamics of the system can be expressed as follows:

$$
\begin{aligned}
\dot{R} & =R \Omega_{\times} \\
\dot{b}_{B} & =R V \\
\dot{P} & =P \times \Omega+\left(m g-F_{B}\right) R^{T} e_{3}+F_{D}+F_{C} \\
\dot{\Pi} & =\Pi \times \Omega+P \times V+m g r_{G} \times R^{T} e_{3}+\mathrm{T}_{D}+\mathrm{T}_{C}
\end{aligned}
$$

The two first lines represent the kinematics of the system while the other two lines describe the dynamics of the vehicle. The term $\left(m g-F_{B}\right) R^{T} e_{3}$ is the contribution of both gravitational and buoyancy forces. The cross term $\operatorname{mgr}_{G} \times$ $R^{T} e_{3}$ expresses the gravitational moment with respect to the center of buoyancy. In any submerged vehicle the center of mass lies under the center of buoyancy, it is straightforward to verify that gravitational moment can be expressed as mgle $_{3} \times R^{T} e_{3}$, where $l$ is the distance between CB and $\mathrm{CG}$, along the body-reference direction $e_{3}^{b}$. This cross term plays an important role in the stabilization of the roll and pitch angles of the vehicle. The expansion of $P \times V$ in Eq. (5), shows that the term $(M V) \times V$ should not be ignored due the added mass of the AUVs [3]. The terms $F_{D}$ and $\mathrm{T}_{D}$ represent the damping terms due to fluid pressure and viscous drag. For underwater vehicles maneuvering at low speed, the damping is given by [1, p.74]:

$$
\left[\begin{array}{ll}
F_{D} & \mathrm{~T}_{D}
\end{array}\right]^{T}=-B(\nu) \nu, \text { with } B(\nu)=B_{l}+B_{n}(\nu)
$$

where $B_{l}$ is a constant diagonal positive matrix and $B_{n}$ represents a nonlinear damping matrix here modeled as $B_{n}=$ $\operatorname{diag}\left(d_{u}|u|, d_{v}|v|, d_{w}|w|, d_{p}|p|, d_{q}|q|, d_{r}|r|\right)$ with $d_{(.)}>0$. We assume hereafter and w.l.o.g that:

$$
F_{D}(V)=-B_{V}(V) V, \quad \mathrm{~T}_{D}(\Omega)=-B_{\Omega}(\Omega) \Omega
$$

where $B_{V}$ and $B_{\Omega}$ are two diagonal positive matrices. Finally, $F_{C}, \mathrm{~T}_{C} \in \mathbb{R}^{3}$ represent the control inputs of the system.

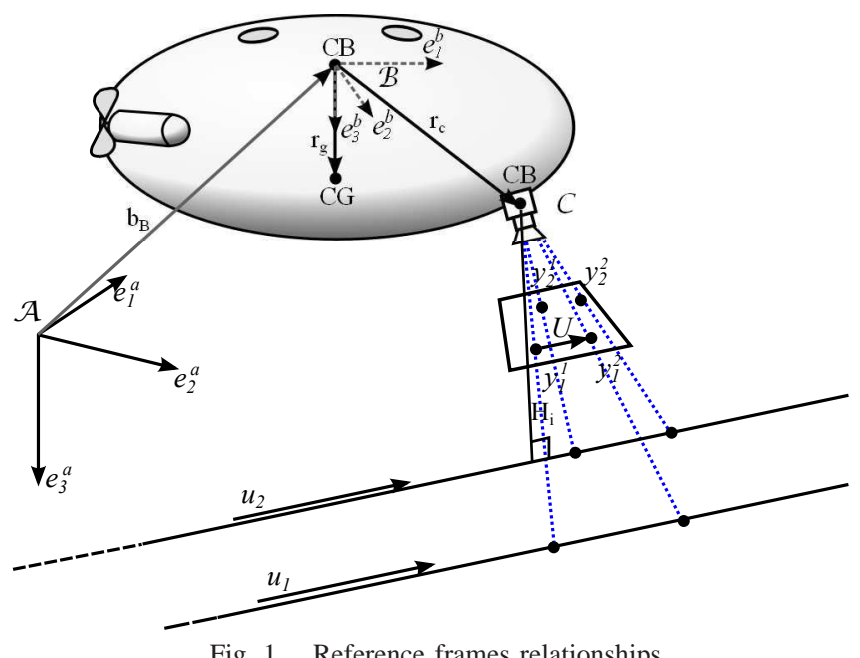

\section{VISUAL SERVO CONTROL PROBLEM}

This section describes the control problem considered to perform autonomous pipeline tracking for fully-actuated 
AUV using visual servo control. The visual features considered are the images of the pipeline borders assumed to be parallel in the horizontal plane of the inertial frame. The unit direction vector of the pipeline is denoted $\mathbf{u} \in \mathcal{A}$ in the inertial frame and $U=R^{T} \mathbf{u} \in \mathcal{C}$ in the camera frame. We assume the image features remain in the camera field of view throughout the motion, a reasonable assumption given the nature of the manoeuvre considered.

A position-like and yaw related orientation error terms are derived from the visual features. The first one is based on the bi-normalised Plücker coordinates of the observed parallel lines and is used to align the camera at a desired height with respect to the pipeline. The second is based on the alignment of the forward velocity with the pipeline direction. Given that the observed lines are known to be parallel, their Plücker coordinates $\left(h_{i}, U\right) \in \mathcal{C}, i=\{1,2\}$ can be measured directly from the image features [17]:

$$
h_{i}=\frac{y_{i}^{1} \times y_{i}^{2}}{\left|y_{i}^{1} \times y_{i}^{2}\right|}=\frac{y_{i}^{1} \times U}{\left|y_{i}^{1} \times U\right|}=\frac{y_{i}^{2} \times U}{\left|y_{i}^{2} \times U\right|} ; U= \pm \frac{h_{1} \times h_{2}}{\left|h_{1} \times h_{2}\right|}
$$

where $y_{i}^{1}$ and $y_{i}^{2} \in \mathcal{C}$ are two specific points on the line $i=$ $\{1,2\}$. The direction of the pipeline $U \in \mathcal{C}$ is specified up to a sign that should be fixed by the operator. The proposed visual servo scheme is based on centroid vectors designed from the image features (8) as follows:

$$
q=h_{1}+h_{2}
$$

The visual position error with respect to the set of the linear features can then be defined as:

$$
\delta_{1}=q-q^{*}
$$

where $q^{*}$ is the value of the $q$ vector for a reference configuration of the features. It is considered as constant in $\mathcal{A}$. Therefore, the dynamics of $q^{*}$ is equivalent to the dynamics of $U: \dot{q}^{*}=-\Omega \times q^{*}$. To regulate the velocity of the vehicle in the direction of the pipeline, a reference velocity $\alpha$ is specified. The yaw related-orientation error can be defined by specifying $e_{1}^{b}$ as the "reference forward direction" for the vehicle. Let define $U_{2}$ as the second component of $U$, one can define the yaw related orientation error by $U_{2} e_{3}$.

Finally, it is worth noting that the external torque due to gravity and buoyancy in a bottom-heavy vehicle (CG is below $\mathrm{CB}$ ) ensures a pendulum-like movement around the gravitational direction $e_{3}^{a}$. Moreover, if the damping is included, the system looses rotational kinetic energy and naturally comes back to equilibrium set point, ensuring that $R^{T} e_{3}$ converges to $e_{3}$ and the first two components of $\Omega$ $\left(\Omega_{1}=p, \Omega_{2}=q\right)$ converge to zero as long as the external torque due to gravity and buoyancy is not compensated.

According to [17], the time derivative of $\delta_{1}$ defined by Eq. (9) can be expressed as follows:

$$
\dot{\delta}_{1}=-\Omega \times \delta_{1}-Q\left(V_{C} \times U\right),
$$

where $V_{C}$ is the velocity of the camera expressed in the body-fixed frame $\mathcal{B}$ and is given by

$$
V_{C}=V-\Omega \times r_{C}
$$

The matrix $\mathrm{Q}$ is symmetric positive definite matrix and is defined by

$$
Q:=\sum_{i=1}^{2} \frac{1}{\left|H_{i}\right|} \pi_{h_{i}}
$$

with $\left|H_{i}\right|$ the norm of the closest point on line $i(i=\{1,2\})$ and $\pi_{x}=\left(I_{3}-x x^{T}\right)$ is a projection onto the tangent space of the sphere $S^{2}$ of a point $x \in S^{2}$. Given that the term $\left|H_{i}\right|$ is unknown it follows that $Q$ is also unknown. In practice, one can use different clues like depth gauge readings or camera autofocus information to estimate the upper-bound and lower-bound of its eigenvalues that will be exploited in the control design. It is of interest to study the structural properties of Eq. (10). The time derivative of the storage function $\frac{1}{2}\left|\delta_{1}\right|^{2}$ is:

$$
\frac{1}{2} \frac{d}{d t}\left|\delta_{1}\right|^{2}=-\delta_{1}^{T} Q\left(V_{C} \times U\right) .
$$

Since the matrix $Q>0$, choosing $V_{C} \times U=\delta_{1}$ acts to decrease $\left|\delta_{1}\right|^{2}$. Analogously, in order to stabilize $U$ about $e_{1}$, consider the following storage positive function

$$
\mathcal{L}_{y}=k_{y}\left(1-U^{T} e_{1}\right), k_{y}>0,
$$

whose time-derivative satisfies

$$
\dot{\mathcal{L}}_{y}=k_{y}\left(\Omega_{\times} U\right)^{T} e_{1}=k_{y} \Omega^{T}\left(U \times e_{1}\right)
$$

To give the control insight, assume that the first two components of $\Omega$ are equal to zero and choose $\Omega_{3}=r$ as

$$
\Omega_{3}=k_{U} U_{2}, k_{U}>0,
$$

One can ensure that $\mathcal{L}_{y}$ is decreasing

$$
\dot{\mathcal{L}}_{y}=-k_{y} k_{U} U_{2}^{2} \text {. }
$$

Since $V_{c}$ and $\Omega_{3}$ cannot be chosen as control input, the approach taken in this paper is split into two parts. The first part termed inner-loop controller allows stabilization the velocities to a desired set point and the second one, termed outer-loop controller, is specifically designed from image feature to define the desired set point.

\section{INNER-LOOP CONTROL DESIGN}

Since the external torque due to gravity and buoyancy ensures a pendulum-like movement around $e_{3}^{a}$, we consider here the objective of stabilizing the velocities $(V, \Omega)$ at the reference $\left(V_{r}, \Omega_{r}\right)$ with $\Omega_{r}=\Omega_{3 r} e_{3}$, defined by the outerloop controller. Let us define the velocity error variables

$$
\widetilde{V}:=V-V_{r}, \quad \widetilde{\Omega}:=\Omega-\Omega_{r} .
$$

Then, the control objective is equivalent to the stabilization of $(\widetilde{V}, \widetilde{\Omega})$ to zero. Using Eqs. (4), (5), and (14), and the fact that $D \Omega_{r}=D \dot{\Omega}_{r}=0$, the velocity error system can be written as

$$
\left\{\begin{aligned}
M \dot{\tilde{V}}-D \dot{\tilde{\Omega}} & =(M V-D \Omega)_{\times} \widetilde{\Omega}+(M \widetilde{V}-D \widetilde{\Omega})_{\times} \Omega_{r} \\
& +F_{r}+\left(m g-F_{B}\right) R^{T} e_{3}+F_{D}(V)+F_{C} \\
I \dot{\widetilde{\Omega}}+D \dot{\tilde{V}} & =(I \Omega+D V)_{\times} \widetilde{\Omega}+(M V-D \Omega)_{\times} \widetilde{V} \\
& +(I \widetilde{\Omega}+D \widetilde{V})_{\times} \Omega_{r}+(M \widetilde{V}-D \widetilde{\Omega})_{\times} V_{r} \\
& +m g l e_{3} \times R^{T} e_{3}+\mathrm{T}_{r}+\mathrm{T}_{D}(\Omega)+\mathrm{T}_{C}
\end{aligned}\right.
$$

with the new variables

$$
\begin{aligned}
& F_{r}:=-M \dot{V}_{r}+\left(M V_{r}\right)_{\times} \Omega_{r} \\
& \mathrm{~T}_{r}:=-I \dot{\Omega}_{r}-D \dot{V}_{r}+\left(I \Omega_{r}+D V_{r}\right)_{\times} \Omega_{r}+\left(M V_{r}\right)_{\times} V_{r}
\end{aligned}
$$


For control design purposes, let us introduce some definitions of saturation functions

$$
\begin{aligned}
\sigma_{\chi}(x) & :=\min (|x|, \chi) \operatorname{sign}(x), \forall x \in \mathbb{R} \\
\operatorname{sat}_{\Delta}(x) & :=\left[\sigma_{\chi_{1}}\left(x_{1}\right), \sigma_{\chi_{2}}\left(x_{2}\right), \sigma_{\chi_{3}}\left(x_{3}\right)\right]^{T}, \forall x \in \mathbb{R}^{3}
\end{aligned}
$$

with a constant positive vector $\Delta=\left[\chi_{1}, \chi_{2}, \chi_{3}\right]$. The first control result of this paper is stated next.

Proposition 1 Let $K_{V}, K_{\Omega}, \Delta_{V}, \Delta_{\Omega}$ denote some positive diagonal matrices and apply the control law

$$
\left\{\begin{aligned}
F_{C}= & -s_{\Delta_{V}}\left(K_{V} \widetilde{V}\right)-(M \widetilde{V})_{\times} \Omega_{r}+M\left(\widetilde{\Omega}_{\times} V_{r}\right) \\
& -F_{r}-\left(m g-F_{B}\right) R^{T} e_{3}-F_{D}\left(V_{r}\right) \\
\mathrm{T}_{C}= & -s_{\Delta_{\Omega}}\left(K_{\Omega} \widetilde{\Omega}\right)-(I \widetilde{\Omega})_{\times} \Omega_{r}+(D \widetilde{\Omega})_{\times} V_{r} \\
& -\mathrm{T}_{r}-\mathrm{T}_{D}\left(\Omega_{r}\right)
\end{aligned}\right.
$$

to the error system (15). Assume that $V_{r}, \Omega_{r}$ and their timederivative are bounded. Then

1) The error state $\left(\widetilde{V}, \widetilde{\Omega}, R^{\top} e_{3}\right)$ converges to $\left(0,0, \pm e_{3}\right)$ for all initial conditions.

2) The equilibrium $\left(\widetilde{V}, \widetilde{\Omega}, R^{\top} e_{3}\right)=\left(0,0, e_{3}\right)$ is locally exponentially stable and the equilibrium $\left(\widetilde{V}, \widetilde{\Omega}, R^{\top} e_{3}\right)=\left(0,0,-e_{3}\right)$ is unstable.

Proof: Consider the following Lyapunov candidate function

$$
\begin{aligned}
& \mathcal{L}_{v e l}=\frac{1}{2} \widetilde{W}^{\top} M_{T} \widetilde{W}+m g l\left(1-e_{3}^{T} R^{T} e_{3}\right) \\
& =\frac{1}{2} \widetilde{V}^{T} M \widetilde{V}+\frac{1}{2} \widetilde{\Omega}^{T} I \widetilde{\Omega}-\widetilde{V}^{T} D \widetilde{\Omega}+m g l\left(1-e_{3}^{T} R^{T} e_{3}\right)
\end{aligned}
$$

with the positive-definite matrix $M_{T}$ defined in Eq. (1). The time-derivative of $\mathcal{L}_{\text {vel }}$ satisfies

$$
\begin{aligned}
\dot{\mathcal{L}}_{v e l} & =\widetilde{V}^{T}(M \dot{\widetilde{V}}-D \dot{\widetilde{\Omega}})+\widetilde{\Omega}^{T}(I \dot{\widetilde{\Omega}}+D \dot{\widetilde{V}})-m g l \Omega^{T} e_{3} \times R^{T} e_{3} \\
& =\widetilde{V}^{T}(M \dot{\widetilde{V}}-D \dot{\widetilde{\Omega}})+\widetilde{\Omega}^{T}(I \dot{\widetilde{\Omega}}+D \dot{\widetilde{V}})-m g l \widetilde{\Omega}^{T} e_{3} \times R^{T} e_{3}
\end{aligned}
$$

Then, using system (15) and the properties $D \Omega_{r}=0$ and $D(a \times b)=(D a)_{\times} b-(D b)_{\times} a, \forall a, b \in \mathbb{R}^{3}$, one has:

$$
\widetilde{V}^{T}(M V-D \Omega)_{\times} \widetilde{\Omega}+\widetilde{\Omega}^{T}(M V-D \Omega)_{\times} \widetilde{V}=0,
$$

and the control law (18) one deduces

$$
\begin{gathered}
\dot{\mathcal{L}}_{v e l}=\widetilde{V}^{T}\left(F_{C}+F_{r}+\left(m g-F_{B}\right) R^{T} e_{3}+F_{D}(V)\right. \\
\left.\quad+(M \widetilde{V}-D \tilde{\Omega})_{\times} \Omega_{r}\right) \\
+\widetilde{\Omega}^{T}\left(\mathrm{~T}_{C}+\mathrm{T}_{r}+\mathrm{T}_{D}(\Omega)\right. \\
\left.\quad+(I \widetilde{\Omega}+D \widetilde{V})_{\times} \Omega_{r}+(M \widetilde{V}-D \widetilde{\Omega})_{\times} V_{r}\right) \\
=\widetilde{V}^{T}\left(F_{C}+F_{r}+\left(m g-F_{B}\right) R^{T} e_{3}+F_{D}(V)\right. \\
\left.\quad+(M \widetilde{V}-D \tilde{\Omega})_{\times} \Omega_{r}-M\left(\widetilde{\Omega}_{\times} V_{r}\right)+D\left(\widetilde{\Omega} \times \Omega_{r}\right)\right) \\
+\widetilde{\Omega}^{T}\left(\mathrm{~T}_{C}+\mathrm{T}_{r}+\mathrm{T}_{D}(\Omega)+(I \tilde{\Omega})_{\times} \Omega_{r}-(D \tilde{\Omega})_{\times} V_{r}\right) \\
=-\widetilde{V}^{T} \text { sat }_{\Delta_{V}}\left(K_{v} \widetilde{V}\right)-\widetilde{\Omega}^{T} \text { sat }_{\Delta_{\Omega}}\left(K_{\Omega} \widetilde{\Omega}\right) \\
+\widetilde{V}^{T}\left(F_{D}\left(\widetilde{V}+V_{r}\right)-F_{D}\left(V_{r}\right)\right)+\widetilde{\Omega}^{T}\left(\mathrm{~T}_{D}\left(\widetilde{\Omega}+\Omega_{r}\right)-\mathrm{T}_{D}\left(\Omega_{r}\right)\right) \\
\leq-\widetilde{V}^{T} \text { sat }_{\Delta_{V}}\left(K_{v} \widetilde{V}\right)-\widetilde{\Omega}^{T} \text { sat }_{\Delta_{\Omega}}\left(K_{\Omega} \widetilde{\Omega}\right)
\end{gathered}
$$

where the last inequality is obtained using the following property: $x(|x+y|(x+y)-|y| y) \geq 0, \forall x, y \in \underset{\widetilde{R}}{\text {. Since } \dot{\mathcal{L}}_{\text {vel }}}$ is negative semi-definite, the velocity errors $\widetilde{V}$ and $\widetilde{\Omega}$ and, thus, the control variables $F_{N}$ and $\mathrm{T}_{N}$ are bounded. From here, one easily verifies the uniform continuity of $\dot{\mathcal{L}}_{v e l}$ via the boundedness of $\ddot{\mathcal{L}}_{v e l}$. By application of Barbalat's lemma, one deduces the convergence of $\dot{\mathcal{L}}_{v e l}$ and, thus, $\widetilde{V}$ and $\widetilde{\Omega}$ to zero. Then, by application of Barbalat's lemma again one deduces the convergence of $\dot{\widetilde{V}}$ and $\dot{\widetilde{\Omega}}$ to zero. Finally, from the last equation in (15) one deduces the convergence of $e_{3} \times R^{T} e_{3}$ to zero which implies that $R^{\top} e_{3}$ converges to either $e_{3}$ or $-e_{3}$. The proof of local exponential stability of the equilibrium $\left(\widetilde{V}, \widetilde{\Omega}, R^{\top} e_{3}\right)=\left(0,0, e_{3}\right)$ and the proof of instability of the equilibrium $\left(\widetilde{V}, \widetilde{\Omega}, R^{\top} e_{3}\right)=\left(0,0,-e_{3}\right)$ are based on the lineazired systems which are not difficult but are omitted here due to space limitation.

Note that in the calculation of $\dot{\mathcal{L}}_{v e l}$, we have developed a string of mathematical manipulations so as to obtain the control law (18) in a simple form which does not contain many terms depending on $\widetilde{V}$. The main reason is that in practice the measurement of the vehicle's translational velocity is more difficult to obtain and is less precise than that of the angular velocity. Note also that the saturation terms in the control law (18) are useful to account for the constraints of the actuators.

Remark 1 The knowledge about the time-derivative of the reference velocities $V_{r}$ and $\Omega_{r}$ are prerequisite to compute the feedback control $F_{C}$ and $\mathrm{T}_{C}$. In the next section, we will show how this fact influences the visual servoing control design.

\section{OUTER-LOOP CONTROLLER: VisUAL SERVO CONTROL DESIGN}

The control design is based directly on the quantities estimated by the visual sensor. The control objective consists in stabilizing the visual error term $\delta_{1}$ about zero, the vector $U$ about $e_{1}$, and $V^{T} U$ about a the reference value $\alpha$.

In view of Eqs. (10), (4), and (5), the system of interest has a cascaded structure. This may lead one to define the reference velocities $V_{r}$ and $\Omega_{r}$ of the inner-loop as functions of $\delta_{1}$ and $U$ respectively to stabilize $(V, \Omega)$ about $\left(V_{r}, \Omega_{r}\right)$. This strategy requires the explicit knowledge of the timederivative of $V_{r}$ which in turn implies that the time-derivative of $\delta_{1}$ must be known. Since the matrix $Q$ is unknown in (10), we introduce a new variable $\delta_{2}$ satisfying the following dynamics:

$$
\dot{\delta}_{2}=-\Omega \times \delta_{2}-k_{1} \delta_{2}+k_{2} \delta_{1}, \delta_{2}(0)=\delta_{1}(0), k_{1,2}>0
$$

Note that $\delta_{2}$ and its time-derivative $\dot{\delta}_{2}$ are known. Analogously to $\delta_{1}(9), \delta_{2}$ is also orthogonal to $U$.

Lemma 2 The vector $\delta_{2}$, solution to system (20), is orthogonal to $U$ for all time $t$.

Proof: The time-derivative of the following Lyapunov candidate function $\mathcal{V}:=1 / 2\left(\delta_{2}^{T} U\right)^{2}$ satisfies

$$
\begin{aligned}
\dot{\mathcal{V}} & =\left(\delta_{2}^{T} U\right)\left(-\delta_{2}^{T}(\Omega \times U)+U^{T}\left(-\Omega \times \delta_{2}-k_{1} \delta_{2}+k_{2} \delta_{1}\right)\right) \\
& =-k_{1}\left(\delta_{2}^{T} U\right)^{2}=-2 k_{1} \mathcal{V}
\end{aligned}
$$

Thus, $\mathcal{V}$ is non-increasing and remains null for all time since $\mathcal{V}(0)=0$. This concludes the proof. 


\section{A. Definitions of reference velocities}

The next step consists in defining the reference velocities $V_{r}$ and $\Omega_{r}$. The following definitions of $V_{r}$ and $\Omega_{r}$ essentially follow the insights given in [17] with few modifications.

Define the reference translational velocity for the camera $V_{C r}$ as

$$
V_{C r}=k_{\delta} U \times \delta_{2}+\alpha U,
$$

with $k_{\delta}$ some positive gain. Intuitively, if $V_{C} \equiv V_{C r}$ and $\Omega \equiv 0$ one has $V^{T} U \equiv \alpha$. This justifies the term $\alpha U$ in the definition (21) of $V_{C r}$. Using Lemma 2 and Eq. (21) one verifies that

$$
V_{C r} \times U:=-k_{\delta}\left(U_{\times}\right)^{2} \delta_{2}=-k_{\delta} \delta_{2}
$$

The reference angular velocity $\Omega_{r}$ is defined based on the heading control objective. In order to stabilize $U$ about $e_{1}$, recall the time derivative (13) of the storage function (12) and choose:

$$
\Omega_{r}:=k_{U} U_{2} e_{3}, k_{U}>0
$$

one obtains

$$
\dot{\mathcal{L}}_{y}=k_{y} \widetilde{\Omega}^{T}\left(U \times e_{1}\right)-k_{y} k_{U} U_{2}^{2} .
$$

Finally, in view of Eq. (11) we define the reference translational velocity $V_{r}$ as

$$
V_{r}:=V_{C r}+\Omega_{r} \times r_{C} .
$$

Note that $\dot{\delta}_{2}$ and $\dot{U}$ are known, hence $\dot{V}_{r}$ and $\dot{\Omega}_{r}$ are also known ${ }^{2}$.

\section{B. Stability analysis}

Since the reference velocities are specified, i.e. Eqs. (24), (22), (21), one can apply the control law (18) proposed in Section IV in order to stabilize $(V, \Omega)$ about $\left(V_{r}, \Omega_{r}\right)$. Using the fact that $\widetilde{V}, \widetilde{\Omega}$ converge to zero and from (12) and (23) it is straightforward to verify that $U_{2}$ converges to zero. Since $R^{T} e_{3}$ converges to $e_{3}$ along with $U_{2}=0$ one can verify that $U$ converges to $e_{1}$. It remains to show the convergence of $\delta_{1}$ and $\delta_{2}$ to zero. Recalling Eqs. (10) and (20), one verifies that:

$$
\left\{\begin{array}{l}
\dot{\delta}_{1}=-k_{\delta} Q \delta_{2}+\epsilon_{\delta_{1}} \\
\dot{\delta}_{2}=k_{2} \delta_{1}-k_{1} \delta_{2}+\epsilon_{\delta_{2}}
\end{array}\right.
$$

with $\epsilon_{\delta_{1}}:=-\Omega_{\times} \delta_{1}-Q\left(\left(\widetilde{V}+\widetilde{\Omega} \times r_{C}\right) \times U\right), \epsilon_{\delta_{2}}:=-\Omega_{\times} \delta_{2}$ The terms $\epsilon_{\delta_{1}}$ and $\varepsilon_{\delta_{2}}$ converge to zero when $\widetilde{V}, \widetilde{\Omega}$, and $U_{2}$ converge to zero. Therefore, they can be treated as singular perturbations of system (25). As a consequence, by application of singular perturbation theory, in order to prove that $\delta_{1}$ and $\delta_{2}$ converge to zero, it suffices to prove the exponential stability of the equilibrium $\left(\delta_{1}, \delta_{2}\right)=(0,0)$ of the zero dynamics of system (25), corresponding to $\epsilon_{\delta_{1}} \equiv$ $\epsilon_{\delta_{2}} \equiv 0$. This zero dynamics is a linear time-varying (LTV) system

$$
\frac{d}{d t}\left[\begin{array}{l}
\delta_{1} \\
\delta_{2}
\end{array}\right]=\underbrace{\left[\begin{array}{cc}
0 & -k_{\delta} Q \\
k_{2} I_{3 \times 3} & -k_{1} I_{3 \times 3}
\end{array}\right]}_{\mathbf{A}(t)}\left[\begin{array}{l}
\delta_{1} \\
\delta_{2}
\end{array}\right] .
$$

\footnotetext{
${ }^{2}$ Due to the space limitation their expressions are not given in the paper.
}

Lemma 3 Consider the LTV system (26). If $Q$ is uniformly bounded and positive definite, and if

$$
\frac{k_{1}^{2}}{k_{2} k_{\delta}}>\min \left(\lambda_{\max }, \frac{\left(\lambda_{\max }-\lambda_{\min }\right)^{2}}{4 \lambda_{\min }}\right)
$$

where $\lambda_{\max }$ and $\lambda_{\min }$ are the upper-bound and lower-bound of the eigenvalues of $Q$, then the equilibrium $\left(\delta_{1}, \delta_{2}\right)=$ $(0,0)$ is exponentially stable.

Proof: Introduce positive constants $p_{1}$ and $p_{2}$ satisfying

$$
p_{1} p_{2}>1,
$$

which serve to build a matrix $\mathbf{P}:=\left[\begin{array}{cc}p_{1} I_{3 \times 3} & -I_{3 \times 3} \\ -I_{3 \times 3} & p_{2} I_{3 \times 3}\end{array}\right]$ (constant, symmetric, positive definite) and matrix $\mathbf{W}(t):=$ $\mathbf{A}(t)^{T} \mathbf{P}+\mathbf{P A}(t)$ with $\mathbf{A}(t)$ defined in (26).

To prove the exponential stability of the LTV system (26), it suffices to show the existence of $p_{1}$ and $p_{2}$ such that

$$
\exists \varepsilon>0, \forall t, \mathbf{W}(t) \leq-\varepsilon I_{6 \times 6}
$$

Note that condition (28) is necessary for $\mathbf{P}$ to be positive definite. One verifies that

$$
\mathbf{W}=\left[\begin{array}{cc}
-2 k_{2} I_{3 \times 3} & -p_{1} k_{\delta} Q+\left(k_{1}+k_{2} p_{2}\right) I_{3 \times 3} \\
-p_{1} k_{\delta} Q+\left(k_{1}+k_{2} p_{2}\right) I_{3 \times 3} & 2 k_{\delta} Q-2 p_{2} k_{1} I_{3 \times 3}
\end{array}\right]
$$

For all $X=\left[\begin{array}{ll}x_{1} & x_{2}\end{array}\right]^{T}$ with $x_{1}, x_{2} \in \mathbb{R}^{3}$, one has

$$
\begin{aligned}
\frac{1}{2} X^{T} \mathbf{W} X= & -k_{2}\left|x_{1}\right|^{2}-x_{2}^{T}\left(p_{2} k_{1}-k_{\delta} Q\right) x_{2} \\
& +\left(k_{1}+p_{2} k_{2}\right) x_{1}^{T} x_{2}-p_{1} k_{\delta} x_{1}^{T} Q x_{2}
\end{aligned}
$$

Let $\lambda_{1}, \lambda_{2}, \lambda_{3}(>0)$ denote the eigenvalues of $Q$. One has $\lambda_{\min } \leq \lambda_{i} \leq \lambda_{\max }$. Since $Q$ is symmetric, there exists a matrix $R_{Q} \in S O(3)$ such that $Q=R_{Q}^{T} \Lambda_{Q} R_{Q}$, with $\Lambda_{Q}=$ $\operatorname{diag}\left(\lambda_{1}, \lambda_{2}, \lambda_{3}\right)$. Denoting $\bar{x}_{i}=R_{Q} x_{i}$ with $i=1,2$, one deduces from Eq. (30) that

$$
\begin{aligned}
\frac{1}{2} X^{T} \mathbf{W} X= & -\sum_{i=1}^{3}\left(k_{2} \bar{x}_{1, i}^{2}+\left(p_{2} k_{1}-k_{\delta} \lambda_{i}\right) \bar{x}_{2, i}^{2}\right. \\
& \left.-\left(k_{1}+p_{2} k_{2}-p_{1} k_{\delta} \lambda_{i}\right) \bar{x}_{1, i} \bar{x}_{2, i}\right)
\end{aligned}
$$

We have to prove that there exists some positive constant $\varepsilon$ such that $X^{T} \mathbf{W} X \leq-\varepsilon|X|^{2}$. From here, the proof proceeds by considering two possible cases. $\triangleright$ Case 1 : Consider the case where $\lambda_{\max } \leq \frac{\left(\lambda_{\max }-\lambda_{\min }\right)^{2}}{4 \lambda_{\min }}$. Eq. (27) implies the existence of a constant $c_{\lambda}>0$ such that

$$
\frac{k_{1}^{2}}{k_{2} k_{\delta}}=\lambda_{\max }+c_{\lambda} \text {. }
$$

From Eq. (31), using Cauchy-Schwartz inequality one ensures that $\mathbf{W} \leq-\varepsilon I_{6 \times 6}$ if for all $i \in\{1,2,3\}$

$$
\begin{aligned}
& \left(k_{1}+p_{2} k_{2}-p_{1} k_{\delta} \lambda_{i}\right)^{2} \leq 4\left(k_{2}-\frac{\varepsilon}{2}\right)\left(p_{2} k_{1}-k_{\delta} \lambda_{i}-\frac{\varepsilon}{2}\right) \\
\Leftrightarrow & F_{i}\left(p_{1}\right):=\left(k_{\delta}^{2} \lambda_{i}^{2}\right) p_{1}^{2}-2 k_{\delta} \lambda_{i}\left(k_{1}+p_{2} k_{2}\right) p_{1} \\
& +\left(k_{1}+p_{2} k_{2}\right)^{2}-4\left(k_{2}-\frac{\varepsilon}{2}\right)\left(p_{2} k_{1}-k_{\delta} \lambda_{i}-\frac{\varepsilon}{2}\right) \leq 0
\end{aligned}
$$

Choose $p_{2}=k_{1} / k_{2}$ and $0<\varepsilon<\min \left(2 k_{2}, 2 k_{\delta} c_{\lambda}\right)$. Then, it is easy to verify from this choice and Eq. (32) that the determinant of $F_{i}\left(p_{1}\right)$ is positive. Thus, inequality (33) is satisfied for all $i \in\{1,2,3\}$ if

$$
\left\{\begin{array}{l}
\max _{\lambda_{i}} p_{1}^{-}\left(\lambda_{i}\right) \leq p_{1} \leq \min _{\lambda_{i}} p_{1}^{+}\left(\lambda_{i}\right) \\
p_{1}^{ \pm}\left(\lambda_{i}\right):=\frac{2 k_{1}}{k_{\delta} \lambda_{i}} \pm \frac{\sqrt{4\left(k_{2}-\varepsilon / 2\right)\left(k_{1}^{2} / k_{2}-k_{\delta} \lambda_{i}-\varepsilon / 2\right)}}{k_{\delta} \lambda_{i}}
\end{array}\right.
$$


It is straightforward to verify that

$$
\left\{\begin{array}{l}
\lim _{\varepsilon \rightarrow 0}\left(\min _{\lambda_{i}} p_{1}^{+}\left(\lambda_{i}\right)\right) \geq \frac{2 k_{2}}{k_{1}-\sqrt{k_{1}^{2}-k_{2} k_{\delta} \lambda_{\max }}}>\frac{k_{2}}{k_{1}}=\frac{1}{p_{2}} \\
\lim _{\varepsilon \rightarrow 0}\left(\max _{\lambda_{i}} p_{1}^{-}\left(\lambda_{i}\right)\right) \leq \frac{2 k_{2}}{k_{1}+\sqrt{k_{1}^{2}-k_{2} k_{\delta} \lambda_{\max }}}
\end{array}\right.
$$

Thus, by continuity there exists some positive threshold $\bar{\varepsilon}_{1}$ close enough to zero and smaller than $\min \left(2 k_{2}, 2 k_{\delta} c_{\lambda}\right)$ such that for all $\varepsilon<\bar{\varepsilon}_{1}$ one has

$$
\min _{\lambda_{i}} p_{1}^{+}\left(\lambda_{i}\right)>\max \left(\max _{\lambda_{i}} p_{1}^{-}\left(\lambda_{i}\right), \frac{1}{p_{2}}\right)
$$

which implies the existence of some constant $p_{1}>0$ such that (28) and (34) are satisfied. This concludes the proof.

$\triangleright$ Case 2 : Consider the case where $\lambda_{\max }>\frac{\left(\lambda_{\max }-\lambda_{\min }\right)^{2}}{4 \lambda_{\min }}$. Assume that one can choose some $p_{1}$ and $p_{2}$ such that

$$
\begin{aligned}
& p_{2} k_{1}>k_{\delta} \lambda_{\max } \\
& k_{1}+p_{2} k_{2} \geq p_{1} k_{\delta} \frac{\lambda_{\max }+\lambda_{\min }}{2}
\end{aligned}
$$

Then, from Eq. (31) and the fact that $\left|x_{i}\right|=\left|\bar{x}_{i}\right|$, with $i=$ 1,2 , one deduces

$$
\begin{aligned}
\frac{1}{2} X^{\top} \mathbf{W} X \leq & -k_{2}\left|x_{1}\right|^{2}-\left(p_{2} k_{1}-k_{\delta} \lambda_{\max }\right)\left|x_{2}\right|^{2} \\
& +\left(k_{1}+p_{2} k_{2}-p_{1} k_{\delta} \lambda_{\min }\right)\left|x_{1}\right|\left|x_{2}\right|
\end{aligned}
$$

From here, using Cauchy-Schwartz inequality one ensures that $\mathbf{W} \leq-\varepsilon I_{6 \times 6}$ if

$$
\begin{aligned}
& \left(k_{1}+p_{2} k_{2}-p_{1} k_{\delta} \lambda_{\min }\right)^{2} \leq 4\left(k_{2}-\frac{\varepsilon}{2}\right)\left(p_{2} k_{1}-k_{\delta} \lambda_{\max }-\frac{\varepsilon}{2}\right) \\
& \Leftrightarrow\left(k_{\delta}^{2} \lambda_{\min }^{2}\right) p_{1}^{2}-2 k_{\delta} \lambda_{\min }\left(k_{1}+p_{2} k_{2}\right) p_{1} \\
& +\left(k_{1}+p_{2} k_{2}\right)^{2}-4\left(k_{2}-\frac{\varepsilon}{2}\right)\left(p_{2} k_{1}-k_{\delta} \lambda_{\max }-\frac{\varepsilon}{2}\right) \leq 0
\end{aligned}
$$

There exists a positive constant $\varepsilon$ close enough to zero such that (37) is satisfied if we have the following strict inequality

$$
\begin{aligned}
G_{1}\left(p_{1}\right) & :=\left(k_{\delta}^{2} \lambda_{\min }^{2}\right) p_{1}^{2}-2 k_{\delta} \lambda_{\min }\left(k_{1}+p_{2} k_{2}\right) p_{1} \\
& +\left(k_{1}+p_{2} k_{2}\right)^{2}-4 k_{2}\left(p_{2} k_{1}-k_{\delta} \lambda_{\max }\right)<0
\end{aligned}
$$

It is easy to verify from Eq. (35) that the determinant of $G_{1}\left(p_{1}\right)$ is positive. Thus, inequality (38) is satisfied if

$$
\left\{\begin{array}{l}
p_{1}^{-} \leq p_{1} \leq p_{1}^{+} \\
p_{1}^{ \pm}:=\frac{k_{1}+p_{2} k_{2}}{k_{\delta} \lambda_{\min }} \pm \frac{\sqrt{4 k_{2}\left(p_{2} k_{1}-k_{\delta} \lambda_{\max }\right)}}{k_{\delta} \lambda_{\min }}
\end{array}\right.
$$

Combining Eqs. (28) and (36) one obtains

$$
\frac{1}{p_{2}}<p_{1} \leq \frac{2\left(k_{1}+p_{2} k_{2}\right)}{k_{\delta}\left(\lambda_{\max }+\lambda_{\min }\right)}
$$

One easily verifies from Eq. (35) that

$$
\frac{1}{p_{2}}<\frac{k_{1}}{k_{\delta} \lambda_{\max }}<\frac{2\left(k_{1}+p_{2} k_{2}\right)}{k_{\delta}\left(\lambda_{\max }+\lambda_{\min }\right)}<p_{1}^{+}
$$

Thus, the existence of some $p_{1}>0$, such that Eqs. (39) and (40) are satisfied, is ensured if

$$
\begin{gathered}
\frac{2\left(k_{1}+p_{2} k_{2}\right)}{k_{\delta}\left(\lambda_{\max }+\lambda_{\min }\right)}>p_{1}^{-} \\
\Leftrightarrow\left(k_{1}+p_{2} k_{2}\right)^{2}\left(\frac{\lambda_{\max }-\lambda_{\min }}{\lambda_{\max }+\lambda_{\min }}\right)^{2}<4 k_{2}\left(p_{2} k_{1}-k_{\delta} \lambda_{\max }\right) \\
\Leftrightarrow G_{2}\left(p_{2}\right):=\left(\frac{\lambda_{\max }-\lambda_{\min }}{\lambda_{\max }+\lambda_{\min }}\right)^{2} k_{2}^{2} p_{2}^{2} \\
-2 k_{1} k_{2}\left(2-\left(\frac{\lambda_{\max }-\lambda_{\min }}{\lambda_{\max }+\lambda_{\min }}\right)^{2}\right) p_{2} \\
+k_{1}^{2}\left(\frac{\lambda_{\max }-\lambda_{\min }}{\lambda_{\max }+\lambda_{\min }}\right)^{2}+4 \kappa_{2} k_{\delta} \lambda_{\max }<0
\end{gathered}
$$

The determinant of $G_{2}\left(p_{2}\right)$ is ensured to be positive from the condition (27). This in turn ensures the existence of some $p_{2}>0$ such that $G_{2}\left(p_{2}\right)<0$. We have proved the existence of some positive constants $p_{1}$ and $p_{2}$ satisfying conditions (28), (35), and (36) such that inequality (29) holds for some positive constant $\varepsilon$. This concludes the proof.

\section{SIMULATION RESULTS}

The controller has been implemented and tested together with the dynamics of a realistic AUV. The simulated vehicle belongs to the family of fully-actuated underwater vehicles which possess full control over all components of the control forces and torques. The physical parameters of the vehicle are shown in Table 1. Two border lines of the tracked pipe stay in a horizontal plane (with $Z=0$ ) in the inertial frame. The direction of the lines $\mathbf{u}$ is parallel to the $X$-axis of the inertial frame (i.e., $\mathbf{u}=e_{1}$ ), where their $Y$-coord are $y_{L 1}=1 \mathrm{~m}$ and $y_{L 2}=3 \mathrm{~m}$. The initial position of the vehicle has been chosen as $b_{B}(0)=[0,-5,7]^{T}, 5 \mathrm{~m}$ aside and $7 \mathrm{~m}$ above the tracked pipe. The initial orientation of the vehicle is $75^{\circ}$ rotated from vector $\mathbf{u}$. The initial roll and pitch angles of the vehicle are null. The reference values, the control gains and parameters are given in lower part of Table 2 .

In order to show the robustness of the method to model errors, a different set of parameters are used in the controller, reflecting usually approximative knowledge of the real AUV dynamics. They are given in the upper part of Table 2

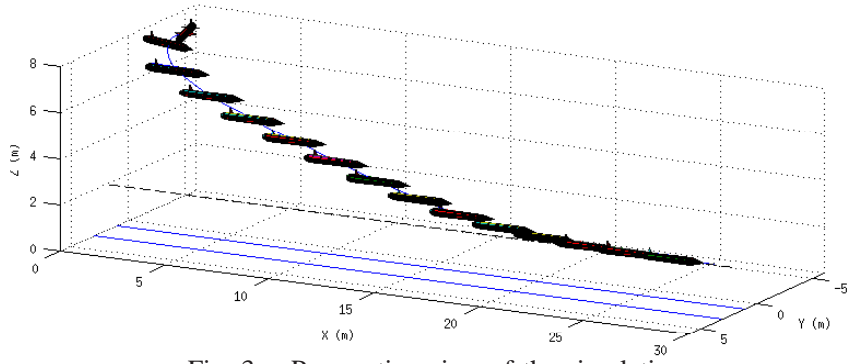

Fig. 3. Perspective view of the simulation

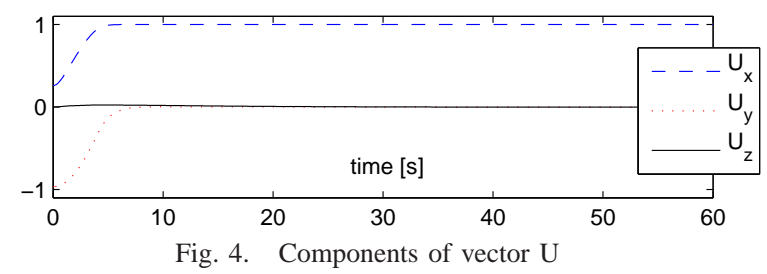

$$
\begin{array}{l|l}
m=1000 \mathrm{~kg} & M_{A}^{11}=10^{3} \operatorname{diag}(0.2,4.5,4.5) \mathrm{kg} \\
\text { volume }=0.97 \mathrm{~m}^{3} & M_{A}^{22}=10^{3} \operatorname{diag}(1.5,20,15) \mathrm{kg} \cdot \mathrm{m}^{2} \\
l=0.15 \mathrm{~m}, r_{C}=[1,0,0]^{T} & B_{V}=10^{3} \operatorname{diag}(0.2,1.0,1.0) \frac{\mathrm{kg}}{\mathrm{g}} \\
B_{\Omega}=10^{5} \operatorname{diag}(0.5,1.2,1.0) \frac{\mathrm{kg}}{\mathrm{s}} \\
I_{0}=\left[\begin{array}{ccc}
500 & -15 & 25 \\
-15 & 3000 & 1 \\
25 & 1 & 3000
\end{array}\right] \mathrm{kg} \cdot \mathrm{m}^{2} & \Delta_{V}=[1000,1000,1000]^{T} \mathrm{~N} \\
& \Delta_{\Omega}=[200,3000,6000]^{T} N
\end{array}
$$

Tab. 1: Physical parameters of the simulated vehicle

The simulation results reported in Fig. 2 show the stabilization of the visual error $\delta_{1}$ to zero, as well as the regulation of the orientation the desired values. The forwarding velocity converges rapidly to the given value $\alpha=0.5 \mathrm{~m} / \mathrm{s}$. The 

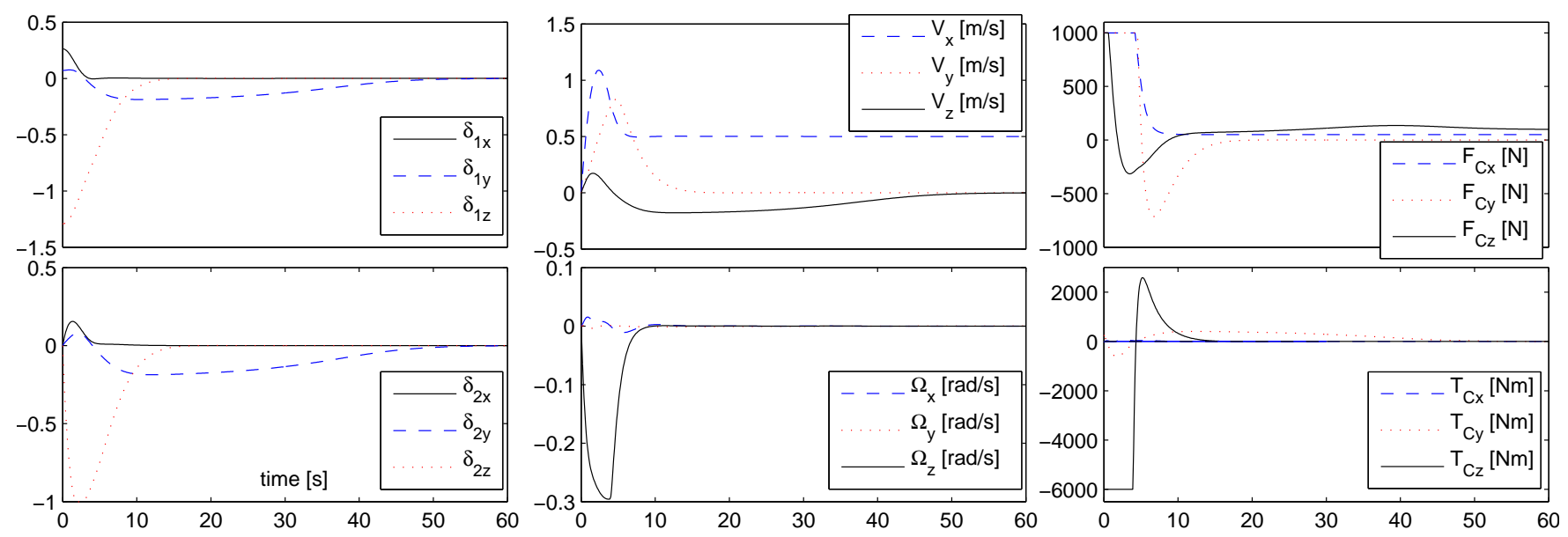

Fig. 2. Numerical results of the simulation. 1st column: error variables of $\delta_{1}$ and $\delta_{2} ;$ 2nd column: body-referenced velocities $V$ and $\Omega$; 3rd column: control forces and torques

\begin{tabular}{|c|c|}
\hline $\begin{array}{l}\hat{I}_{0}=\operatorname{diag}(550,3200,3300) \mathrm{kg} \cdot \mathrm{m}^{2} \\
\hat{\Delta}_{V}=[1100,1100,1100]^{T} N \\
\hat{\Delta}_{\Omega}=[250,3100,6100]^{T} N\end{array}$ & $\begin{array}{l}\hat{M}_{A}^{11}=10^{3} \operatorname{diag}(0.18,4.3,4.4) \mathrm{kg} \\
\hat{M}_{A}^{22} \\
10^{3} \operatorname{diag}(1.6,18,14) \mathrm{kg} \cdot \mathrm{m}^{2}\end{array}=$ \\
\hline $\begin{array}{l}k_{1}=1, k_{2}=1 \\
K_{V}=M, K_{\Omega}=I\end{array}$ & $\begin{array}{l}k_{\delta}=1, k_{U}=1 \\
\alpha=0.5 \frac{m}{s}, q *=[0,1.79,0]^{T}\end{array}$ \\
\hline
\end{tabular}

Tab. 2: Estimated vehicule parameters, desired values and simulation gain settings

vehicle does not experience large pitch and roll angular velocities during the transient period, while only the yaw angular velocity has a visible transient before reaching zero.

The control saturation occured during a short period and did not significantly affect the overall performance of the controller. The values of $\Delta_{V}$ and $\Delta_{\Omega}$ reflect a realistic thrust that real-life propellers could have, with a visible handicap in the pitch regulation - which is the case on several existing AUVs, where pitch is regulated by a separate system, eg. underwater gliders [18]. Thus, the saturation variables can prevent the controller from setting the actuator output to unrealistic or dangerous levels.

\section{CONCLUSIONS}

In this paper we developed a nonlinear visual servo control for tracking linear pipelines by fully-actuated underwater vehicle. More precisely, the controller directly uses the image features as feedback information without explicit of the relative pose of the vehicle with respect to the environment. The stability of the closed-loop system is proved and the control algorithm has been tested within a realistic simulation framework. Certain properties of the real-world vehicles which are not part of the generalized approach taken in the theoretical section can be exploited for further refining of the control scheme. Notably, typical AUV hull often resembles a torpedo shape which implies that one of the principal dimensions is significantly bigger than the others. Associated streamlining also causes that one of the coefficients both of the added mass $\left(M_{A}\right)$ and the form drag $(B)$ are of a different order of magnitude than the others. This can be exploited to further enhance the mathematical expression of the controller. The implementation of this scheme on a real system is now envisaged.

\section{REFERENCES}

[1] T. I. Fossen, Marine Control Systems. Marine Cybernetix AS, 2002.

[2] P. Bailey, W. Price, and P. Temarel, "A unified mathematical model describing the maneuvering of a ship travelling in a seaway," Transactions The Royal Institution of Naval Architects, pp. 131-149, 1997.

[3] N. E. Leonard, "Stability of a bottom-heavy underwater vehicle," Automatica, vol. 33, no. 3, pp. 331-246, 1997.

[4] J. Yuh, "Design and control of autonomous underwater robots: A survey," Autonomous Robot, vol. 8, no. 1, pp. 7-24, 2000.

[5] L. Lapierre, D. Soetanto, and A. Pascoal, "Robust nonlinear pathfollowing control of an AUV," IEEE Journal of Oceanic Engineering, vol. 33, pp. 89-102, april 2008.

[6] T. M. Josserand, "Optimally-robust nonlinear control of a class of robotic underwater vehicles," december 2006.

[7] W. Naeem, R. Sutton, and S. M. Ahmad, "LQG / LTR control of an AUV using hybrid guidance law," in Proceedings of GCUV Conference, april 2003.

[8] D. Fryxell, P. Oliveira, A. Pascoal, C. Silvestre, and I. Kaminer, "Navigation, guidance and control of AUVs: An application to the MARIUS vehicle," Control Engineering Practice, vol. 4, pp. 401-409, march 1996.

[9] I. Spangelo and O. Egeland, "Trajectory planning and collision avoidance for underwater vehicles using optimal control," IEEE Journal of Oceanic Engineering, vol. 19, pp. 502-511, october 1994.

[10] J. Aulinas, C. S. Lee, J. Salvi, and Y. R. Petillot, "Submapping SLAM based on acoustic data from a 6-DOF AUV," in 8th IFAC Conference on Control Applications in Marine Systems, september 2010.

[11] T. Palmer, "A short range vision based navigation system for autonomous docking on subsea intervention panels," june 2008.

[12] F. Maurelli, S. Krupinski, Y. Petillot, and J. Salvi, "A particle filter approach for AUV localization," in Proc. of MTS/IEEE OCEANS, 2008.

[13] P. K. Paim, B. Jouvencel, and L. Lapierre, "A reactive control approach for pipeline inspection with an AUV," in Proceedings of MTS/IEEE OCEANS, vol. 1, 2005, pp. 201-206.

[14] P. Rives and J.-J. Borrelly, "Underwater pipe inspection task using visual servoing techniques," in Proceedings of ICRA, 1997.

[15] Julius Plücker, "On a new geometry of space," Philosophical Transactions of the Royal Society of London, vol. 155, pp. 725-791, 1865.

[16] H. Lamb, Hydrodynamics. Cambridge University Press, 1932.

[17] R. Mahony and T. Hamel, "Image-based visual servo control of aerial robotic systems using linear image features," IEEE Transactions on Robotics and Automation, vol. 21, no. 2, pp. 227-239, April 2005.

[18] J. G. Graver and N. E. Leonard, "Underwater glider dynamics and control," in 12th Int. Symp. on Unmanned Untethered Submersible Techn., 2001. 